\title{
La comunicación institucional en la gestión universitaria: estudio aplicado a la captación de estudiantes
}

\author{
Eva MARTIN-Fuentes \\ Universidad de Lleida \\ eva@aegern.udl.cat \\ Natalia DARIES-RAMÓN \\ Universidad de Lleida \\ ndaries@aegern.udl.cat \\ Eduard CRISTÓBAL FrANSI \\ Universidad de Lleida \\ ecristobal@aegern.udl.cat
}

\begin{abstract}
Resumen:
Este estudio se centra en el análisis de la campaña de comunicación diseñada por la Universidad de Lleida (UdL), para promocionar el grado de Turismo. Se realizó una investigación para obtener el perfil del alumnado del grado de Turismo con la intención de conocer las necesidades y expectativas respecto a los estudios superiores y sus fuentes de información para la elección de sus estudios universitarios, incluyendo tanto Internet como las Redes Sociales. Entre los principales resultados se destaca la importante presencia de la web institucional de la UdL como fuente de información primaria.
\end{abstract}

Palabras clave: campaña de comunicación; publicidad institucional; plan de medios; comportamiento del consumidor; comunicación integrada

\section{Institutional communication in university management: study applied to student recruitment}

\begin{abstract}
:
This study focuses on the analysis of the communication campaign designed by the University of Lleida $(\mathrm{UdL})$ to promote the tourism degree. An investigation was conducted to obtain the profile of the tourism degree students in order to know their needs and expectations about higher education and their sources to choose university, including both Internet and Social Networks. Among the main results we observed a significant presence of institutional web of UdL as a primary information source.
\end{abstract}

Key Words: communication campaign; institutional advertising; media plan; consumer behavior; integrated communication

Referencia normalizada:

Martin Fuentes E., Daries Ramón, N. y Cristóbal Fransi, E. (2014): La comunicación institucional en la gestión universitaria: estudio aplicado a la captación de estudiantes. Historia y Comunicación Social. Vol. 19. Núm. Especial Febrero. Págs. 591-604.

Sumario: 1. Introducción. 2. Marco Teórico. 3. Proceso de Investigación. 4. Análisis de la competencia y del público objetivo. 5. Campaña de comunicación. 6. Resultados de las acciones. 7. Conclusiones. 8. Bibliografía. 9. Anexo: Encuesta 


\section{Introducción}

El trabajo que se presenta a continuación analiza la campaña de comunicación diseñada por la Universidad de Lleida (UdL), para promocionar y dar a conocer el grado de Turismo, que se implantó en el curso 2010/11.

Hasta el curso 2010/11 los estudios de turismo en la ciudad de Lleida, habían sido unos estudios privados que, a través de la diplomatura de turismo, se empezaron a impartir en Lleida el año 1981 en un centro privado.

Es en el curso 2010/11 cuando se inicia el grado de Turismo en la Facultad de Derecho y Economía (FDE) de la UdL. Con una duración de cuatro cursos académicos, se singulariza por un plan de estudios con un enfoque diferenciado, por la relevancia que se da a la formación en gestión empresarial, la introducción de las Nuevas Tecnologías como eje conductor de la mayoría de las asignaturas y la Emprendeduría, dado que la mayor parte de los titulados desarrollarán su carrera profesional en la empresa turística. A nivel pedagógico se apuesta por la evaluación continuada, las metodologías transversales y activas, y la orientación profesionalizadora (con prácticas obligatorias de empresa) a la vez que se inicia a los estudiantes en la investigación (con un trabajo de final de grado).

Desde el decanato de la Facultad, y durante el primer curso del grado de Turismo se vio la necesidad de dar a conocer estos estudios. Para ello se realizó una investigación con la intención de conocer las necesidades y expectativas respecto a estos estudios superiores por parte de los estudiantes. Esta investigación se dividió en dos líneas principales. La primera se basó en un análisis de la competencia y la segunda consistió en la administración de una encuesta en la que se buscaba obtener el perfil del alumnado del grado de Turismo, los motivos de su elección, conocer las fuentes de información que influenciaban a la hora de tomar la decisión y los medios de comunicación a los que acudían, incluyendo entre estos, a los medios virtuales tanto Internet como las redes sociales. Una vez recogida esta información, se trabajó para el diseño de una campaña de comunicación, con el principal objetivo de aumentar la notoriedad de los estudios y captar el máximo número posible de estudiantes para consolidar la titulación del grado de Turismo

Este estudio está estructurado de la siguiente manera: a continuación de esta introducción, se encuentra el marco teórico destacando los principales estudios sobre este tema. La tercera sección describe el proceso de la investigación y en la cuarta se presentan los resultados de análisis de la competencia y el perfil de los estudiantes de turismo de la UdL para conocer sus preferencias antes de llevar a cabo la campaña de comunicación que se explica en la quinta sección. Por último, se presentan los resultados de la campaña de comunicación y, finalmente, las diferentes conclusiones que surgen de este estudio. 


\section{Marco teórico}

La universidad pública española ha experimentado durante los últimos años profundas transformaciones que están motivando cambios importantes respecto al grado de rivalidad soportada, el tipo de actividades desarrolladas y la forma a través de la cual éstas son llevadas a cabo (Lozano y Flavián, 2004). La educación universitaria ha pasado de ser un servicio dirigido a una minoría de la población a convertirse en un ámbito competitivo en la mayor parte de los países desarrollados y por tanto, las universidades tienen que competir por los estudiantes en los diferentes mercados de reclutamiento (Comm y Labay, 1996; Landrum et al., 1998; Luque y el Barrio, 2007). Esto es especialmente cierto en España donde el número de alumnos universitarios se ha visto reducido por el descenso de la natalidad y por las dificultades económicas derivadas de la crisis actual. La demanda ha disminuido, mientras que la oferta no sólo ha aumentado sino que se ha diversificado (Fayos et al., 2011).

Por tanto, los estudiantes y sus familias tienen una gran variedad de opciones entre las que elegir y deben tomar complejas decisiones para realizar la elección correcta (Maringe, 2006). Podemos considerar la elección de los estudios universitarios como lo que se entiende por "decisiones de alta implicación" (Veloutsou et al., 2004).

La utilización del marketing y sus herramientas será la base para que la universidad española pueda competir en el mercado y alcanzar el éxito (Carmelo y Calvo, 2009). Para ello, el uso de los medios de comunicación por parte de las instituciones universitarias es básico para difundir y ayudar en la elección de sus estudios (Alameda y Fernández Blanco, 2005). No sólo se deben utilizar los medios tradicionales sino que la presencia activa tanto en Internet como en las redes sociales se hace imprescindible ya que su uso se ha incrementado rápidamente en predomino y popularidad en los últimos años, especialmente entre los alumnos universitarios (Pempek et al., 2009; Vrocharidou y Efthymiou, 2012).

Hay numerosos estudios que analizan el uso y el efecto de las redes sociales en el estudiantado universitario. Una de las redes de más popularidad entre los jóvenes es Facebook y ésta ha sido objeto de diversas investigaciones desde diferentes perspectivas (Cheung et al., 2011; Hew, 2011; Green y Bailey, 2010; Pempek et al., 2009). Entre las razones que empujan a la participación de los alumnos en Facebook se encuentran las siguientes: comunicarse con amigos, mirar o subir fotos, ocio y entretenimiento, buscar y planear eventos, enviar o recibir mensajes, crear o leer mensajes en el muro, conocer mejor a otros individuos, obtener información de contacto y presentarse por medio del contenido del perfil (Pempek et al., 2009). Si nos centramos en el uso de las redes sociales en el ámbito educativo encontramos estudios como los de Cheung et al. (2011); Green y Bailey (2010); Roblyer et al. (2010) y Teclehaimanot y Hickman, (2011) entre otros. En cambio, Turan et al. (2012), han investigado las razones por las que los estudiantes universitarios no utilizan estas redes sociales, llegando a la conclusión de que los principales motivos eran que se percibían como una pérdida de tiempo, o una herramienta innecesaria; las posibili- 
dades de poder conllevar una adicción; violar las normas de privacidad, compartir información excesiva; e invocar la preocupación parental.

\section{Proceso de investigación}

Con la idea de diferenciar los estudios de grado de turismo recién implantados, adaptados al Espacio Europeo de Educación Superior y organizados desde la Universidad de Lleida y para aumentar la notoriedad de los estudios y captar posibles estudiantes para consolidar esta titulación, se realizó una investigación para disponer de información suficiente con el fin de elaborar una campaña de comunicación para promocionar los nuevos estudios de Turismo.

En primer lugar se llevó a cabo un análisis del entorno, del producto, de la competencia y del público objetivo para poder llevar a cabo la estrategia a seguir y para poder realizar la planificación de la campaña de comunicación.

La información relativa al entorno, producto y competencia se obtuvo a partir de datos secundarios consultados a través de los sitios web de las diferentes universidades y del Departamento responsable de universidades del gobierno de Cataluña.

Para conocer el público objetivo, se analizó el perfil de los estudiantes que acababan de iniciar los estudios del grado de Turismo y se obtuvo algunos datos a través de la propia Universidad de Lleida (vías de acceso a estos estudios, ramas de enseñanzas de ciclo formativo de grado superior de procedencia, edades, procedencia geográfica, entre otros).

Una vez el planteamiento estaba claro y realizadas las técnicas de tipo exploratorio, como son las discusiones de grupo, entrevistas no dirigidas, observación, y las fuentes de información secundarias externas Por otro lado, se elaboró una encuesta (Anexo 1) en la que se plantearon distintas preguntas a los estudiantes matriculados para conocer su perfil: los motivos de la elección de estos estudios en la UdL, conocer las fuentes de información y de prescripción que influyen a la hora de tomar la decisión y los medios de comunicación a los que acuden, y otras cuestiones relativas a su comportamiento ante las redes sociales y la satisfacción lograda hasta el momento con estos estudios. Asimismo, se plantearon preguntas abiertas sobre aspectos positivos y aspectos negativos de la carrera, para mejorar en lo que fuera posible y conveniente los estudios del Grado de Turismo en la UdL. Algunas de las preguntas de la encuesta se basaron en la encuesta que realiza la Universidad de Lleida a todos los estudiantes de primer curso, pero adaptadas a estos estudios.

Esta encuesta se administró entre el 22 y el 28 de noviembre de 2010 y se analizó durante el mes de diciembre de 2010. 


\section{Análisis de la competencia y del público objetivo}

Los principales resultados de la investigación se muestran a continuación.

\subsection{Análisis de la competencia}

Los estudios de grado de turismo de la Universidad de Lleida tenían como competidores directos los mismos estudios impartidos por otras universidades cercanas geográficamente.

Por una parte, las universidades que ofrecían estos estudios de forma oficial como la Universidad Rovira y Virgili (URV) y la Universidad de Girona (UdG) representaban sus principales competidores, seguido de las universidades del área metropolitana de Barcelona que ofrecían los estudios de turismo pero de forma privada. También era competidora de la Universidad de Lleida, las 120 plazas ofrecidas en Zaragoza del grado de turismo y las del campus de Huesca, todas ellas de la Universidad de Zaragoza. Este competidor podía ser especialmente importante, para dejar de captar los estudiantes que venían de los pueblos de Aragón limítrofes a la provincia de Lleida.

Como competidores indirectos encontrábamos también los ciclos formativos de grado superior enfocados en el ámbito del turismo y la hostelería y otras titulaciones universitarias vinculadas al ámbito de la geografía y la empresa impartidas por la propia Universidad de Lleida o por otras universidades.

Los estudios de turismo de la Universidad Abierta de Cataluña (UOC) también eran competidores directos de los de la UdL aunque el perfil de estudiante de turismo en la UOC era diferente al del de la UdL. Partiendo del informe sobre la evaluación de los estudios de economía y empresa de la UOC realizado por la AQU (se parte de estos estudios porque no existe en la AQU la evaluación de los estudios de turismo), el perfil de sus estudiantes es en un $80 \%$ adulto entre 25 y 40 años, en más de un $90 \%$ con empleo y en un $22 \%$ con otra titulación y en un $43 \%$ con titulación sin finalizar (AQU, 2011)

Respecto al tipo de estudiantado podemos afirmar que los estudios de grado de Turismo de la UdL en el curso 2010/11 acogieron un total de 32 estudiantes y su perfil eran jóvenes entre 17 y 25 años, de los cuales el 53\% era mujeres y el $47 \%$ hombres. Procedían en un $41 \%$ de la ciudad de Lleida, un $47 \%$ de la provincia de Lleida (38\% de las comarcas de la Plana de Lleida y $9 \%$ de las comarcas del Pirineo de Lleida) y el $12 \%$ restante de Barcelona. Las vías de acceso fueron un $82 \%$ de bachillerato, un $9 \%$ de ciclos formativos de grado superior y el $9 \%$ de otras vías como la de mayores 40 años y de otras universidades, según información facilitada por la UdL (2011).

\subsection{Fuentes de información de los estudios de turismo}

Los estudiantes se informaban principalmente a través de la página web de la UdL. En menor grado, por las tutorías en el instituto y de información aportada por amigos o familiares, y también se destacaba la información obtenida en ferias como el Salón 
de la Enseñanza de Barcelona y en menor medida en las Jornadas de Campus Abiertos y en las sesiones en los institutos organizadas por la UdL. Nadie afirmó haberse informado a través de noticias de la prensa y tan sólo una persona lo hizo a través de anuncios insertados en los diarios locales.

Figura 1. Información de los estudios

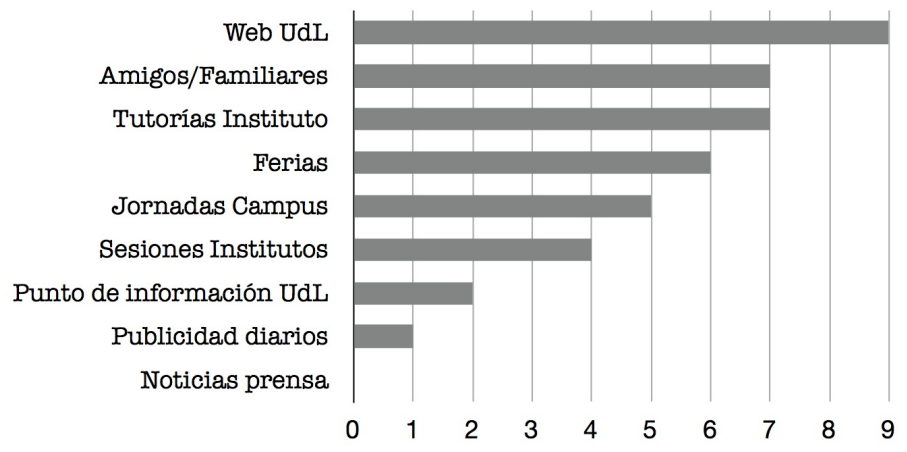

Fuente: Elaboración propia

\subsection{Motivación por escoger los estudios de turismo}

La principal motivación por haber escogido los estudios de turismo era porque les gustaba viajar y los idiomas. El siguiente motivo era porque no habían podido entrar a su primera opción que era en cinco casos educación infantil y primaria, en dos casos INEFC y en un caso Psicología, Derecho o Comunicación Audiovisual. También consideraban importante como motivación que se trataba de unos estudios con futuro profesional y, finalmente, porque les gustaban estos estudios, trabajaban en el sector o tenían un negocio familiar.

Figura 2. Motivación elección estudios

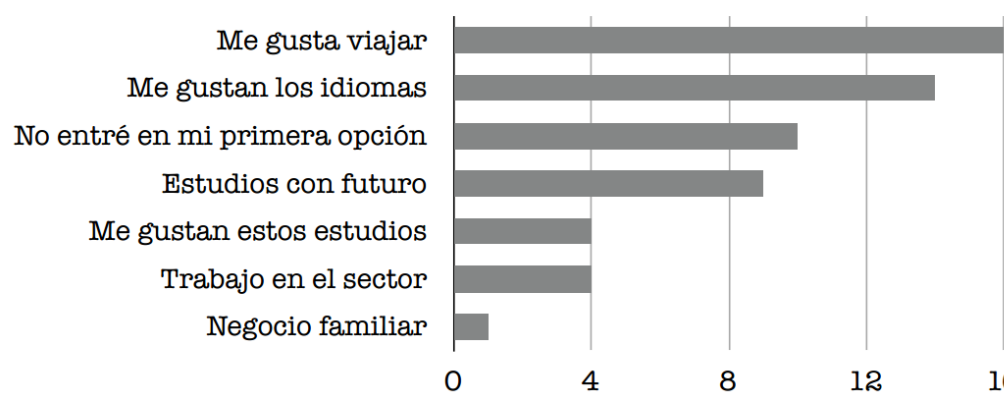

Fuente: Elaboración propia 
4.4 Motivación por escoger los estudios en la UDL

La principal motivación para escoger los estudios de Turismo en la Universidad de Lleida era por proximidad geográfica, en segundo lugar porque la consideraban una buena universidad y por el plan de estudios que ofrecía.

Figura 3. Motivación elección universidad

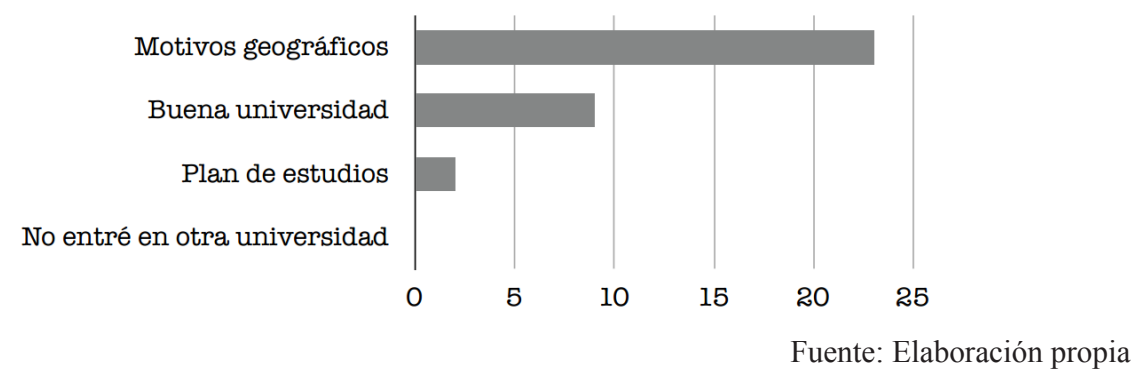

4.5 Uso de redes sociales

A la pregunta sobre si eran usuarios de redes sociales, todos ellos, excepto un $8 \%$ usaban alguna red social. De los usuarios, un 91\% lo eran de Facebook, un 4,5\% de Tuenti y un $4,5 \%$ de Twitter.

\subsection{Música}

Todos los estudiantes de Turismo de la UdL afirmaban escuchar música, un $81 \%$ lo hacían a través de Internet y un $44 \%$ a través de la radio.

La música por internet la escuchaban principalmente a través de Spotify, aplicación empleada para la reproducción de música vía streaming y la música de la radio a través de la emisora local Flaix FM.

\section{Campaña de comunicación}

Con toda la información obtenida se planteó una campaña de comunicación en el que el sitio web de la UdL debía tener un papel importante dado que era la principal fuente de información para los estudiantes y se recomendaba que estuviera siempre actualizada, que tuviera los programas de todas las asignaturas. Asimismo, se recomendaba aprovechar las acciones de promoción de la propia UdL que hacía en general, y no sólo de estos estudios, como las tutorías en los centros, la participación en ferias o las jornadas de campus abiertos. 
Dado que los estudiantes que escogían los estudios de turismo lo hacían porque les gustaba viajar y los idiomas, la propuesta de mensaje de comunicación debía girar en torno a estos elementos y se planteó el siguiente mensaje:

Sube al barco del turismo, vuela tus estudios, habla las lenguas de la aventura, pedalea tu futuro, camina tus ilusiones.

Matricúlate en el Grado de Turismo de la Universidad de Lleida.

Tu viaje profesional comienza con el Grado de Turismo de la Universidad de Lleida, no pierdas esta oportunidad. El Grado de Turismo una opción de futuro. Entra en la web www.turisme.udl.cat

El público objetivo era usuario de redes sociales, especialmente de Facebook por lo que se propuso utilizar este canal para llegar a ellos. Al no ser posible gestionar una cuenta de Facebook sólo para los estudios de turismo, se optó por pagar publicidad en esta red social ya que permitía segmentar por edad, población, gustos, entre otros.

El público objetivo escuchaba música por Internet a través de Spotify y por la radio a través de Flaix FM, por lo que se optó con poner cuñas en estos dos medios con el mensaje descrito anteriormente.

Se desaconsejó insertar publicidad en prensa escrita local porque era costosa y no parecía que se hicieran demasiado eco a través de este medio.

Se observaba también que habían accedido pocos estudiantes de ciclos formativos de grado superior, por lo que se recomendó hacer acciones de información concretas para intentar captar este público.

El presupuesto de la campaña se distribuyó un $67 \%$ en Spotify, un $22 \%$ en la cadena de radio Flaix FM, un 6\% para la publicidad en Facebook y un 5\% se destinó para la creatividad (mensaje, grabación y diseño del bánner). Las acciones se realizaron entre el mes de mayo y los primeros días de junio de 2011, coincidiendo con unos días previos y durante la preinscripción universitaria.

Figura 4. Bánner difundido en Facebook y Spotify

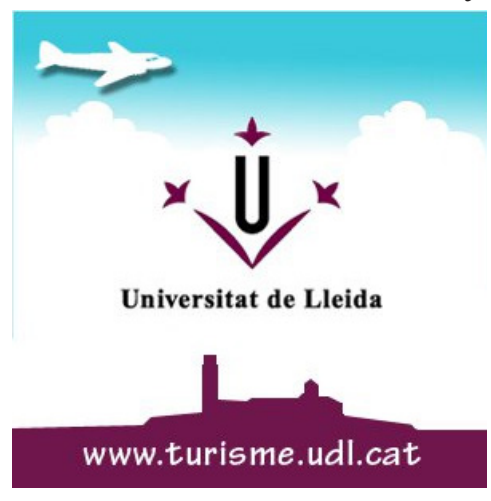


A partir de una reflexión profunda sobre el análisis de resultados de la campaña de comunicación de las dos primeras promociones, y de un estudio pormenorizado de las experiencias de los profesores implicados en el Grado de Turismo, se consideró que el alcance de la campaña debía ser más integrador y global. Para conseguirlo se realizaron acciones en la propia universidad a nivel interno, en el proceso de aprendizaje, que formaron parte de la estrategia de comunicación global, para ello se implantó en la titulación un Proyecto de Innovación Docente y se organizaron las I Jornadas de Nuevas Tecnologías y Turismo

Los objetivos de estas acciones se centraron en contribuir a la difusión, intercambio y extensión de las experiencias de mejora e innovación docente y acercar el mundo real y laboral a la universidad.

\section{Resultados de las acciones}

El anuncio en Spotify obtuvo 125.127 impresiones, 380 clics a la web www. turisme.udl.cat y un CTR (Click-through rate) del 0,30\%. El anuncio en Facebook obtuvo 1.679.692 impresiones, 337 clics a la web www.turisme.udl.cat y un CTR del $0,02 \%$. Las cuñas en la cadena Flaix FM, al no ser un medio digital, no se pudo medir los resultados de forma directa. Un año después, se volvió a preguntar a los estudiantes que ese curso 2011/12 habían optado por matricularse a primero del grado de Turismo en la UdL si habían escuchado el anuncio. En esa encuesta, de los 39 estudiantes de primero la contestaron un total de 26 . El 27\% afirmaba haber escuchado el anuncio de Spotify o de Flaix FM y un 11,5\% indicaba que las campañas en Spotify o Flaix FM les había animado a escoger los estudios de Turismo de la UdL.

Con las estrategias realizadas a nivel interno, se logró mejorar la relación universidad empresas, conociendo las necesidades reales de los empresarios del entorno de Lleida, y una gran repercusión en los medios de comunicación locales de Lleida: prensa, radio, televisión y en las redes sociales.

\section{Conclusiones}

En el tercer año de la implantación de los estudios de grado de Turismo de la Universidad de Lleida, se puede afirmar que la campaña de comunicación ha sido fructífera ya que en el primer año sin campaña de comunicación se inscribieron un total de 32 personas, en el segundo año se inscribieron 39 y en el tercero 47, repitiendo el último año, la misma campaña de comunicación con menor presupuesto pero con mayor implicación de profesorado y estudiantes, y realizando más acciones internas. 
Se observa que, a parte de la comunicación que realiza cada universidad de todos sus estudios, es beneficioso realizar acciones de promoción más focalizadas para cada titulación ya que consideramos que son más productivas al permitir alcanzar de forma más directa al público objetivo, especialmente porque el mensaje puede ser más concreto. Las acciones generales de promoción de las universidades son positivas, por lo que se recomienda aprovecharlas para reforzar el mensaje.

Los estudiantes usan el sitio web institucional de la UdL como fuente de información primaria por lo que se recomienda que esté siempre actualizada y que tenga toda la información que puedan necesitar los futuros estudiantes, especialmente los programas de todas las asignaturas para conocer sus contenidos.

Se destaca que el mensaje llegó a los estudiantes a través de la cadena de radio Flaix FM y de la aplicación Spotify pero en cambio, no recibieron el mensaje a través de Facebook.

Gracias a la repercusión de la campaña y los comentarios en las redes sociales, en los blogs de profesionales y entidades de turismo y las herramientas de publicity, se ha conseguido promover y dar a conocer la implantación del Grado de Turismo y mejorar la relación con los profesionales y los empresarios del ámbito turístico de Lleida que en un futuro serán los demandantes de los futuros profesionales que actualmente se están formando.

Esta investigación podría servir como base para planificar campañas de comunicación a otras universidades que imparten los estudios de turismo a un público con perfil similar al que se presenta en este estudio.

\section{Bibliografía}

AQU (2011). Agencia de Calidad de la Generalitat de Cataluña. Evaluación de los estudios de Economía y Empresa (UOC). Disponible en: http://www.aqu.cat/doc/ doc_42878623_1.pdf. [5-09-2013]

ALAMEDA D.; FERNÁNDEZ-BLANCO, E. (2005). Comunicación La comunicación publicitaria de las universidades españolas. En DE SALAS et al. (2005). La universidad en la comunicación, la comunicación en la universidad. p. 263-272. Madrid: Colección Comunicación 2000.

CARMELO M.; CALVO S. (2009). Branding Universitario: Marcando la Diferencia. Madrid: Editorial Delta.

CHEUNG, C.M.K.; CHIU, P.Y.; LEE, M.K.O. (2011). "Online Social Networks: Why do Stu-dents Use Facebook?". Computers in Human Behavior, 27(4), p. 1337-1343.

COMM, C.L.; LABAY, D.G. (1996). "Repositioning colleges using changing student quality perceptions: An exploratory analysis". Journal of Marketing for Higher Education, Vol.7 No.4, p. 21-35. 
FAYOS, T.; GONZALEZ-GALLARZA, M.; SERVERA D.; ARTEAGA F. (2011). "Análisis y evaluación del servicio de formación universitaria: implicaciones para el marketing estratégico de las universidades". Revista de Investigación en Educación, $\mathrm{N}^{\mathrm{o}}$ 9, Vol. 2. p. 1-28.

GREEN, B.T.; BAILEY, B. (2010). “Academic Uses of Facebook: Endless Possibilities or Endless Perils?". TechTrends, 54(3), p. 20-22.

HEW, K.F. (2011). "Students' and Teachers' Use of Facebook". Computers in Human Behavior, 27, p. 662-676.

LANDRUM, R.E.; TURRISI, R.; HARLESS, C (1998). "University image: the benefits of assessment and modeling". Journal of Marketing for Higher Education, Vol.9 No.1, p. 53-68.

LOZANO F. J.; FLAVIÁN C. (2004). "La orientación al mercado de la Universidad Pública: un reto para el sistema universitario español". Revista internacional de marketing público y no lucrativo. Vol. 1, $\mathrm{N}^{\circ}$. 2, p. 9-28.

LUQUE, T.; DEL BARRIO, S. (2007). "Análisis del valor de las percepciones de los clientes en el diagnóstico estratégico de la universidad". Ponencia presentada en el 6 th International Congress Marketing Trends, 26-27 de enero. París.

MARINGE, F. (2006). "University \& Course Choice: Implications for positioning, recruitment and marketing". International Journal of Educational Management, Vol.20 No.6, p. 466-479.

PEMPEK, T.; YERMOLAYEVA, A.Y.; CALVERT, L.S. (2009). "College Students' Social Network-ing Experiences on Facebook". Journal of Applied Developmental Psychology, 30. p. 227-238.

ROBLYER, M.D.; MCDANIEL, M.; WEBB, M.; HERMAN, J.; WITTY, J.V. (2010). "Findings on Facebook in Higher Education: A Comparison of College Faculty and Student Uses and Perceptions of Social Networking Sites". The Internet and Higher Education, 13(3), p. 134-140.

TECLEHAIMANOT, B.B.; HICKMAN, T. (2011). "Student-teacher Interaction on Facebook: What Students Find Appropriate". TechTrends, 55(3), p. 19-30.

TURAN Z.; TINMAZ H.; GOKTAS Y. (2013). "The Reasons for Non-Use of Social Networking Websites by University Students". Scientific Journal of Media Education. $\mathrm{N}^{\circ}$. 41. p. 1-15.

VELOUTSOU, C.; LEWIS, J.W.; PATON, R.A. (2004). "University selection: information requirements and importance". International Journal of Educational Management, Vol.18 No.3, p. 160-171.

VROCHARIDOU, A.; EFTHYMIOU, I. (2012). "Computer Mediated Communication for Social and Academic Purposes: Profiles of Use and University Students' Gratifications". Computers \& Education, 58(1), p. 609-616. 


\section{Notas}

9.1. Encuesta al primer curso del grado de turismo de la UDL

¿Qué estudiabas el año pasado?

Bachillerato. Centro

Ciclo Formativo de Grado Superior. Indica especialidad y centro

No estudiaba

Otros. Indica

Antes de entrar en la UdL, ¿dónde recibiste información de los estudios de turismo de la UdL?

$\square$ Actividades de tutoría en el instituto

Jornadas de Campus Abiertos en la UdL

Salón de la Enseñanza

Punto de información de la UdL

Sesiones informativas de la UdL al instituto

Web de la UdL

Anuncios en los diarios

Noticias en los medios de comunicación

A través de amigos o familiares

Otros. Indica cuáles:

Antes de entrar en la UdL, ¿conocías la diferencia entre el grado de turismo de la UdL y la diplomatura que se imparte en la Escuela de Turismo "Terres de Lleida"?

$\square$ Sí, antes de entrar en la UdL, ya sabía que la Escuela de Turismo era privada y que los estudios eran de 3 años (plan antiguo no adaptado al Espacio Europeo de Educación Superior)

$\square$ No, no conocía la diferencia

¿Por qué has elegido estudiar turismo?

$\square$ Me gustan los idiomas

$\square$ Me gusta viajar

$\square$ Son los estudios que más me gustan

Por negocio familiar

Trabajo actualmente en el sector

Creo que son unos estudios con mucho futuro

No pude entrar en mi primera opción que era

Otras. Indica el motivo

¿Por qué has elegido estudiar turismo en la UdL?

$\square$ Motivos geográficos

Es una buena universidad

No entré en otra universidad que era

Por el plan de estudios que ofrece

Otros. Indica el motivo

¿La semana de acogida te ayudó a integrarte en la UdL?

Sí, completamente

Sí, pero me faltó información. Indica cuál:

No, no me sirvió de nada

No pude asistir. Indica el motivo:

¿Tienes intención de acabar estos estudios en la UdL?

$\square$ Sí 
$\square$ No, quiero continuar estos estudios en otra universidad

$\square$ No, quiero cambiar de estudios

¿Tienes pensado participar en algún programa de movilidad mientras haces la carrera (Erasmus, Seneca-Sicue, , etc.)?

$\square$ Sí, me gustaría marchar una temporada a estudiar fuera

$\square$ No, no quiero marchar

$\square$ Todavía no lo sé

¿Recomendarías a tus amistades y/o familiares los estudios de turismo a la UdL?

$\checkmark$ Sí. ¿Por qué?

$\square$ No. ¿Por qué?

¿Eres usuario de redes sociales?

$\square$ Sí, Facebook

$\square$ Sí, Tuenti

$\square$ Sí, otros. Indica cuál

$\square$ No

¿Escuchas música?

$\square$ Sí, por internet. ¿Spotify? Sí / No ¿Otros? cuál:

$\square$ Sí, en la radio. ¿Qué emisora?

$\square$ No

Valora tu grado de acuerdo con estos afirmaciones

(1: muy en desacuerdo, 5: muy de acuerdo)

\begin{tabular}{|c|c|c|c|c|c|}
\hline $\begin{array}{l}\text { La orientación de los profesores ha sido importante para escoger los } \\
\text { estudios de turismo }\end{array}$ & 1 & 2 & 3 & 4 & 5 \\
\hline $\begin{array}{l}\text { La orientación de los padres y/o familiares ha sido importante para escoger } \\
\text { los estudios de turismo }\end{array}$ & 1 & 2 & 3 & 4 & 5 \\
\hline $\begin{array}{l}\text { La opinión de los amigos y compañeros ha sido importante para escoger } \\
\text { los estudios de turismo }\end{array}$ & 1 & 2 & 3 & 4 & 5 \\
\hline $\begin{array}{l}\text { Mi opinión personal, sin tener en cuenta la de los otros, ha sido importante } \\
\text { para escoger los estudios de turismo }\end{array}$ & 1 & 2 & 3 & 4 & 5 \\
\hline $\begin{array}{l}\text { La información sobre los estudios de turismo de la web de la UdL me ha } \\
\text { sido útil }\end{array}$ & 1 & 2 & 3 & $\overline{4}$ & 5 \\
\hline $\begin{array}{l}\text { La información recibida sobre la UdL en el centro donde he estudiado ha } \\
\text { sido adecuada y útil }\end{array}$ & 1 & 2 & 3 & 4 & 5 \\
\hline Estoy contento y satisfecho de estudiar turismo en la UdL & 1 & 2 & 3 & 4 & 5 \\
\hline $\begin{array}{l}\text { Estoy vinculado a la vida universitaria (consejos del estudiantado, cargos } \\
\text { de representación, asociaciones, etc.) }\end{array}$ & 1 & 2 & 3 & 4 & 5 \\
\hline
\end{tabular}

Los estudios de turismo en la UdL, ¿han cumplido tus expectativas?

$\square$ Sí

$\square$ No

Indica lo que más te gusta de los estudios de turismo en la UdL

Indica qué se podría mejorar de los estudios de turismo en la UdL 


\section{Los autores}

Eva Martin-Fuentes. Diplomada en Turismo, Licenciada en Publicidad y Relaciones Públicas y Master en Dirección y Planificación del Turismo. Profesora colaboradora y Secretaria académica del departamento de Administración de Empresas en la UdL.

Su investigación está centrada en el impacto económico de los eventos y en el impacto de las nuevas tecnologías en el turismo. Ha participado en congresos nacionales e internacionales con artículos sobre la contribución de eventos en el turismo de negocios, impacto de las nuevas tecnologías y sobre promoción turística y redes sociales.

Natalia Daries-Ramón. Diplomada en Turismo, Licenciada en Administración y Dirección de Empresas, Licenciada en Investigación y Técnicas de Mercado y Master en Sociedad de la Información y el Conocimiento. Profesora colaboradora y Coordinadora del Grado de Turismo en la UdL.

Su investigación está centrada en el impacto y la implantación de las nuevas tecnologías en el turismo. Ha participado en congresos nacionales e internacionales con artículos sobre el impacto de las nuevas tecnologías y las redes sociales en destinos turísticos.

Eduard Cristóbal Fransi. Doctor en Economía por la Universidad de Lleida (UdL). Profesor del área de comercialización e investigación de mercados. Director del departamento de Administración de Empresas en la UdL. Su investigación está centrada en el comercio electrónico, implantación de las TIC y la evaluación de la calidad percibida. Ha participado en congresos nacionales e internacionales y publicado en revistas como International Journal of Market Research, International Journal of Marketing Studies, International Journal of Business and Management, International Journal of Services Technology and Management and Managing Service Quality, y ha colaborado en diferentes libros como "E-Commerce and V-Business: Digital Enterprise in the Twenty-First Century" o "Social E-Enterprise: Value Creation through ICT" entre otros. 